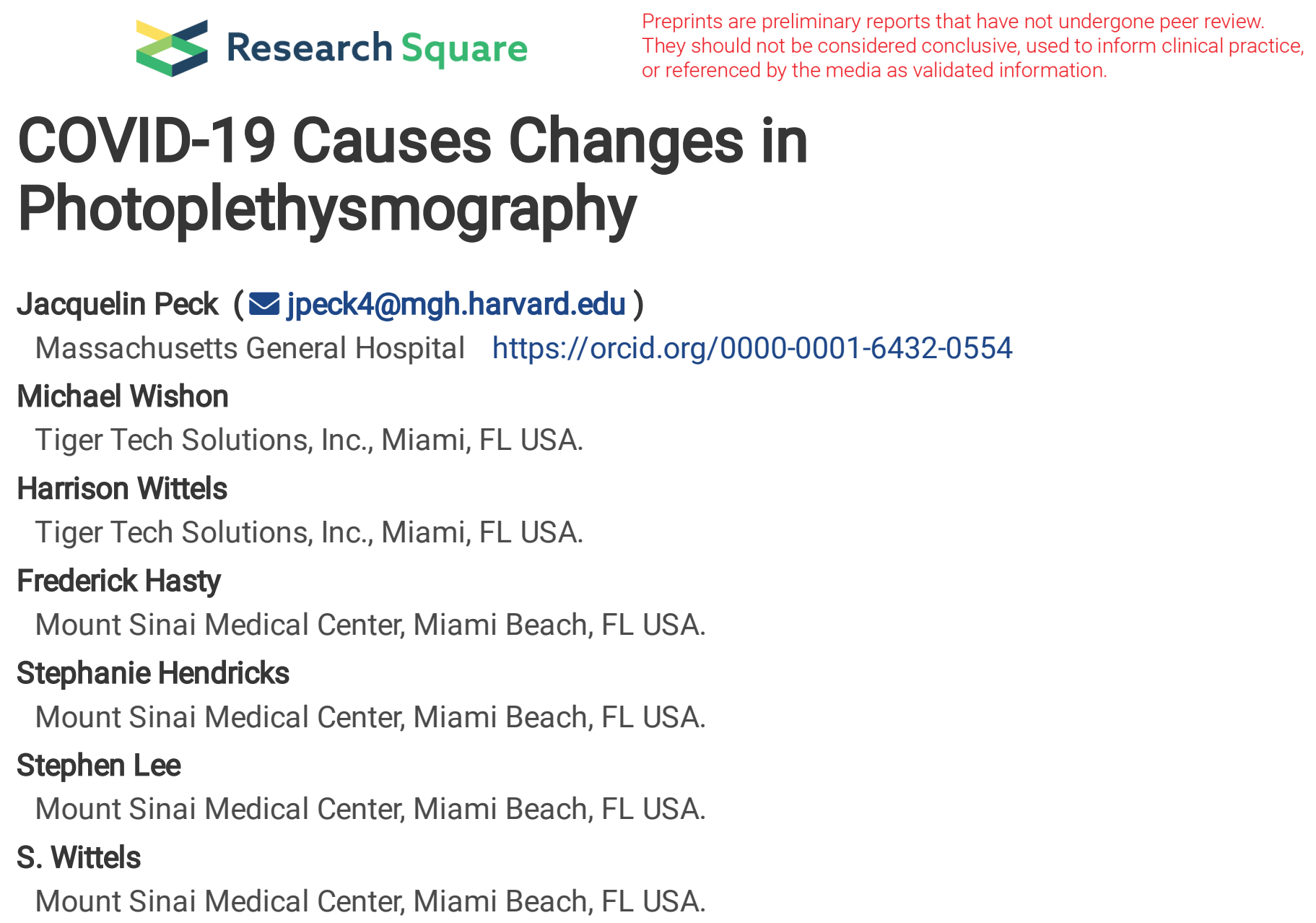

Tiger Tech Solutions, Inc., Miami, FL USA.

\section{Frederick Hasty}

Mount Sinai Medical Center, Miami Beach, FL USA.

\section{Stephanie Hendricks}

Mount Sinai Medical Center, Miami Beach, FL USA.

\section{Stephen Lee}

Mount Sinai Medical Center, Miami Beach, FL USA.

\section{S. Wittels}

Mount Sinai Medical Center, Miami Beach, FL USA.

\title{
Article
}

Keywords: COVID-19, Photoplethysmography (PPG), oximeter

Posted Date: September 29th, 2021

DOl: https://doi.org/10.21203/rs.3.rs-899550/v1

License: (c) (1) This work is licensed under a Creative Commons Attribution 4.0 International License. Read Full License 


\section{Abstract}

Photoplethysmography (PPG) is the science behind many commonly used medical devices such as the pulse oximeter. PPG dropouts have been described in existing in vitro studies following artificially induced clot activation. Because COVID-19 causes increased arterial, venous and microvascular clot formation, our hypothesis is that PPG dropout identified in vitro can also be found in vivo in patients with COVID-19. To test this hypothesis, we prospectively collected PPG recordings and d-dimer levels for 197 ICU patients with COVID-19 and compared them with the PPG tracings from 300 non-COVID controls.

Results demonstrated significant differences in rates of PPG dropout between patients with COVID-19 vs non-COVID controls. The median PPG dropout rate was 0.58 for COVID-19 patients (median 0.58, IQR 0.42-0.72, p<0.05) as opposed to a median 0.0 for non-COVID patients (median 0.0, IQR 0.0-0.0, p<0.05). Further, at least one incidence of PPG dropout was detected in $100 \%$ of COVID-19 patients, as opposed to $2.3 \%$ of non-COVID controls $(p<0.05)$.

PPG dropout also correlated closely with normalized serum d-dimer levels taken on the same day. Ddimer is an established biomarker used for COVID-19 disease severity and prognosis. The change in normalized d-dimer was plotted against the change in PPG dropout and a line of best fit was created. Linear regression resulted in $R^{2}=0.919(p<0.05)$, indicating that PPG dropout rate correlates with hemorheological changes in COVID-19 patients.

The difference in the median and IQR PPG dropout values for patients with vs. without COVID-19 and the strong correlation with serum d-dimer shows the potential utility of such a metric for identifying individuals with acute hemorheological abnormalities associated with COVID-19.

\section{Introduction}

Hemorheology is the study of hematologic flow properties. Alterations in hemorheology or blood flow mechanics can be seen in many disease processes especially relating to infection, inflammation, sepsis, atherosclerosis, and cardiovascular disease (1). COVID-19 (or SARS-Co-V2) is a global pandemic responsible for more than four million deaths globally since it was first reported in 2019 (2). COVID-19 was once thought to be a primarily respiratory disease but is now better understood as a systemic inflammatory disease with well-established hemorheological changes. These changes include inflammation-induced hyperviscosity, hypercoagulability, hyperfibrinoginemia, and thrombosis that may be refractory to therapeutic anticoagulation $(3,4)$. One recent evaluation of COVID-19 induced hyperviscosity using capillary viscometry reported significantly increased plasma viscosity ranging from 1.9-4.2 centipoise (reference range 1.4-1.8) as well as increased fibrinogen concentrations ranging from $459-1188 \mathrm{mg} / \mathrm{dL}$ (median value $708 \mathrm{mg} / \mathrm{dL}$; normal reference range $200-393 \mathrm{mg} / \mathrm{dL}$ ).

Hypercoagulability and thrombosis increase COVID-19 morbidity have led to complications including deep vein thrombosis, pulmonary embolism, and stroke (5). 
Real-time monitoring of hemorheological changes may help inform clinical decision making. However, modern assessments of hemorheology have many limitations. Advanced hemorheological testing such as Lumi aggregometry, light transmission, platelet aggregation, and flow cytometry is primarily reserved for advanced specialty centers and research labs (6). Serum D-dimer is a well-established marker for COVID-19 disease severity and prognosis $(7,8)$. However, serum D-dimer and other commonly used laboratory measurements of platelet adhesion, clotting and degradation, blood viscosity, and rigidity index are invasive, nursing labor intensive, and may take several hours to provide results. Furthermore, frequent lab draws contribute to iatrogenic anemia and increase costs. Finally, increasingly popular pointof-care testing options such as the thromboelastogram (TEG) are expensive and require a high level of training and expertise to perform and interpret results. Moreover, TEG is time consuming to perform and is a static measure of hemorheology at the time of blood draw, indicating that the acquired information may be obsolete by the time lab results are available or that repeated measures may be necessary for rapidly evolving clinical scenarios.

In a 2017 Scientific Reports publication, Njoum et al evaluated the accuracy of photoplethysmography (PPG) as a non-invasive, diagnostic measure of hemorheology in an in vitro model mimicking human arterial circulation (6). The study used PPG, an intra-arterial pressure transducer, and an ultrasound doppler flowmeter to track changes in flow mechanics following alterations in shear rate and clot formation (6). PPG AC and PPG DC both achieved statistically significant amplitude changes during periods of increased shear rate and immediately following thromboplastin activation. The authors also described a change in PPG AC morphology and amplitude following clot formation. The authors concluded that photoplethysmography could be used as an effective, non-invasive method for mapping hemorheology and identifying patterns in blood flow mechanics, fibrin cross-linking, and clot disaggregation in vitro (6).

The following is a proof-of-concept study applying PPG findings reported by Njoum et al to living patients for the first time. In this study, we evaluate PPG tracings from 197 patients with COVID-19 and compare them to PPG tracings from 300 non-COVID controls. We also compare PPG trends within the COVID-19positive population to trends in serum D-dimer, an established clinical marker for COVID-19 $(7,8)$. PPG recordings were obtained using a USFDA-authorized COVID-19 armband screening device (TigerTech CovidPlus $^{\mathrm{TM}}$ monitor) (9). If significant, PPG changes may offer a dynamic, non-invasive, and affordable alternative to existing COVID-19 measures.

\section{Methods}

\section{Study design and participants}

This is a partially prospective, observational, single-institution study. A total of 497 patients were enrolled (300 COVID-19-negative, and 197 COVID-19-positive). Inclusion criteria for the prospectively collected COVID-19 cohort included COVID-19-positive status with confirmatory SARS-CoV-2 RT-PCR and Intensive Care Unit (ICU) admission within our institution's ICU from April 8, 2020, to June 29, 2020. Exclusion 
criteria included age less than 18.0 years or ICU admission without a SARS-CoV-2 RT-PCR positive test result.

A control group of 300 COVID-19-negative patients was retrospectively selected from an institutional database consisting of 6000 patients with PPG recordings and were assumed to be COVID-19 negative because the data was collected prior to the first reported case of COVID-19 within the United States (10). Patient selection was completed using a random number generator in MATLAB. Exclusion criteria included age less than 18.0 years. No exclusion criteria based on disease status or comorbidity were applied. The non-COVID cohort was not matched to the COVID-19 positive cohort.

All PPG recordings were assessed for PPG peaks, pulse rate, PPG dropouts, and dropout rate. This study was approved by our institution's Internal Review Board (Federalwide Assurance Number:

FWA00000176).

\section{Data collection}

Included patients' electronic medical records were reviewed by trained, IRB-approved physicians. COVID19 status, demographic information, and lab results were recorded. PPG waveforms from 197 COVID-19positive ICU patients were prospectively recorded by a CITI trained research coordinator. Recordings were time-stamped and saved for analysis. D-dimer levels and SARS-CoV-2 RT-PCR were prospectively obtained by study physicians from the patients' electronic medical records. 300 non-COVID patients were randomly selected from an institutional database for comparison and corresponding PPG recordings and demographic information were retrospectively collected. D-dimer values were not available for the nonCOVID cohort due to retrospective design.

\section{Photoplethysmography monitoring}

PPG recordings were obtained using a TigerTech CovidPlus Monitor ${ }^{\text {TM }}$ (TigerTech Solutions, Inc. Miami, FL), which is a USFDA-authorized COVID-19 armband screening device with Red and IR PPG capabilities. Acquired data was analyzed using a proprietary algorithm to detect PPG peaks and PPG dropouts (9).

PPG peaks are defined as the point of maximal amplitude for each pulse wave from the continuous PPG recording. Utilizing PPG peaks, pulse rate is calculated as the number of pulse peaks that occurred in 1 minute for a pulse rate of pulses per minute (PPM). PPG dropouts are defined as a binary metric where during any single heartbeat or, PPG peak-to-peak pulse wave, the PPG signal voltage value decreased such that it is below the PPG dropout threshold. The PPG dropout threshold was defined as the 60 second moving average of the PPG signal minus the 60 second moving standard deviation of the PPG signal. For every pulse wave there is either a dropout or not depending on whether the condition is met or not. Using PPG dropouts and pulse rate, a ratio of PPG dropouts per pulse wave or dropout rate was derived as the ratio of number of dropouts per minute divided by pulse rate in beats per minute (number 
of PPG dropouts per minute / pulse waves per minute). This gives a ratio of dropouts / pulse wave as a number between 0 and 1 (with 0 corresponding to a dropout in $0 \%$ of pulse waves and with 1 corresponding to a dropout in $100 \%$ of pulse waves).

\section{Statistical analysis}

Statistical significance was set at $p<0.05$. Two sample t-tests were performed. Analysis was performed using linear regression analysis and comparing the above statistical metrics between COVID and nonCOVID patients utilizing scatter and line plots.

\section{Role of the Funding Source}

Armband PPG recording were obtained using the Tiger Tech Warfighter Monitor ${ }^{\mathrm{T}}$. The photoplethysmography device used in this study (TigerTech Warfighter Monitor ${ }^{\mathrm{TM}}$ ) was provided by TigerTech Solutions Inc. The Tiger Tech Warfighter Monitor ${ }^{\mathrm{TM}}$ was developed partially under a SBIR contract (W81XWH19C0059) from the Defense Health Agency. However, no funding was provided from Tiger Tech Solutions, Inc. or from the Defense Health Agency for the present post-development study using the device.

\section{Results}

\section{Demographics}

In this section, photoplethysmography (PPG) tracings from 497 patients are analyzed and presented (197 COVID-19 positive ICU patients; 300 non-COVID controls). Relevant medical information was recorded for the duration of their hospitalizations. The randomly selected control group was representative of the COVID-19 dataset in terms of age, sex, ethnicity, and comorbidities. The COVID-19 positive cohort and the control cohort had similar ages (median 67 years [IQR: $54.75-79.25]$ vs. 59 years [IQR: $35.5-82.5]$ ), gender distribution ( $59 \%$ male vs. $47 \%$ male), race ( $41 \%$ vs. $27 \%$ Hispanic, $39 \%$ vs. $47 \%$ Caucasian, and $19 \%$ vs. $18 \%$ African American), obesity ( $49 \%$ vs. $38 \%$ ), diabetes ( $21 \%$ vs $12 \%)$, and heart rate ( $75[62-88]$ vs. 83[IQR: 74-92]). PPG recordings were obtained for an average of 3.4 minutes [IQR 3.1-4.2], but all samples were cut to 3 minutes in duration for analysis.

\section{Photoplethysmography Dropout}

To quantify PPG dropout, the 60-second moving mean and 60-second moving standard deviation based on IR PPG data was calculated for each subject in the study. The dropout threshold was calculated as the moving mean minus the moving standard deviation of the signal. A dropout was then defined as any excursion, during a single pulse wave, of the PPG signal below the dropout threshold. A sample PPG 
tracing from a COVID-19-negative patient is displayed in Figure 1. A red dot-dash line denotes the dropout threshold. To regularize the data, the bias was removed, and the PPG data was normalized by dividing by 1.65 (11). In this figure, no PPG voltage values are smaller than the red dashed line and therefore there are zero PPG dropout events within this sample. Conversely, a sample COVID-19 patient PPG recording is provided in Figure 2. A red dot-dash line again denotes the dropout threshold. Here, every pulse wave has a value which extends below the threshold (red dashed line), indicating a PPG dropout event for every pulse wave within this sample.

\section{Photoplethysmography Dropout Rate}

The rate of PPG dropout events was calculated for each patient as a ratio of PPG dropouts per pulse wave for the total number of pulse waves per PPG recording. As an example, a PPG dropout rate of 1.00 indicates that $100 \%$ of pulse waves within a given PPG recording resulted in a PPG dropout and a PPG dropout rate of 0.00 indicates that $0 \%$ of pulse waves within a given PPG tracing resulted in a PPG dropout. The PPG dropout rate was calculated for each patient and trends in dropout rates for COVID-19 positive and non-COVID patients are presented in Table 1. Non-COVID control patients exhibited a median PPG dropout rate of zero (median PPG Dropout Rate: 0; IQR 0.00-0.00, $p<0.05$ ). Patients with COVID-19 exhibited a mean PPG dropout rate of 0.58 (median PPG Dropout Rate: 0.58; IQR 0.42-0.72, p<0.05).

Maximum and minimum PPG dropout rates are also provided in Table 1. Of the 300 non-COVID patients, seven (2.3\%) experienced a PPG dropout in at least one pulse wave. Among non-COVID patients, the lowest recorded PPG dropout rate was 0.00 and the maximum recorded PPG dropout rate was 0.01 . This indicates that the highest PPG dropout rate demonstrated by a non-COVID patient was one PPG dropout per one-hundred pulse waves. Conversely, of the 197 COVID-19-positive patients, 197 (100\%) experienced PPG dropout in one or more pulse waves. Among patients with COVID-19, the lowest recorded PPG dropout rate was 0.11 and the maximum recorded PPG dropout rate was 1.00. This indicates that the lowest PPG dropout rate displayed by a patient with COVID-19 was eleven PPG dropouts per one-hundred pulse waves and the highest PPG dropout rate displayed by a COVID patient was one-hundred PPG dropouts per one-hundred pulse waves. These results reached statistical significance.

Table 1

PPG Dropout Rates among COVID-19-Positive and non-COVID Patients 


\begin{tabular}{|llll|}
\hline COVID-19 Status & COVID-19 Positive $(n=197)$ & COVID-19 Negative $(n=300)$ & $p$-value \\
\hline Dropout Rates: median (IQR) & $0.58(0.42-0.72)$ & $0.00(0.00-0.00)$ & $<0.05$ \\
\hline Number with dropouts $(\%)$ & $197 / 197(100.0 \%)$ & $7 / 300(2.3 \%)$ & - \\
\hline Maximum dropout rate & 1.00 & 0.01 & - \\
\hline Minimum dropout rate & 0.11 & 0.00 & - \\
\hline
\end{tabular}

\section{Photoplethysmography and D-dimer}

D-dimer is an established biophysical marker for COVID-19-positive disease progression and severity $(7,8))$. We therefore trended PPG dropout rates and compared PPG dropout rates to trends in serum Ddimer levels for all included COVID-19-positive patients. Of the 197 COVID-19 positive patients, 21 patients had seven or more consecutive days of d-dimer lab values available and therefore had adequate data for d-dimer trend analysis. The remaining patients were discharged or expired within 7 days of admission. Figure 3 displays a sample trend of serum d-dimer protein level and PPG drop rate plotted over time. This figure, which is statistically similar to the other 20 , shows a strong temporal correlation between PPG drop rate and levels of serum D-dimer. The PPG data in the figures were normalized by 1.65 and the DC bias was removed. The D-Dimer levels were normalized by dividing all the levels by the maximum D-Dimer level acquired for that patient during their stay.

Figure 4 is a scatter plot comparing trends in d-dimer change to trends in PPG dropout rate change. There are 197 points on the scatter plot, one point representing each patient with COVID-19. For each patient, the normalized percent change in d-dimer for the patient's hospitalization (patient's maximum d-dimer value minus minimum d-dimer value divided by maximum d-dimer during hospitalization) was plotted against the corresponding change in PPG dropout rate for PPG recordings taken on the dates of maximum and minimum d-dimer levels (PPG dropout rate from date of maximum d-dimer level minus the PPG dropout rate from the date of the minimum d-dimer) for all 197 COVID-19 patients with at least two d-dimer and PPG data points. The data was then analyzed using linear regression which resulted in an R2 $=0.919(p<0.05)$, demonstrating a strong correlation between changes in D-dimer level and changes in PPG dropout rates in COVID-19 patients.

\section{Discussion}

COVID-19 infects human hosts using the angiotensin converting enzyme type 2 (ACE2) (12). ACE2 is expressed throughout the body in organs like the lungs, heart, intestines, kidneys, and is also expressed intravascularly by endothelial cells (12). A growing body of research points to endotheliitis as a cause of hemorheological changes and a key component of COVID-19 pathophysiology (13-16). Under normal conditions, endothelial cells help prevent coagulation within the intravascular space by producing 
heparan sulphate proteoglycans within the glycocalyx; by expressing thrombomodulin, which binds to thrombin and limits its affinity for fibrinogen and other coagulation factors; by expressing endothelial protein $\mathrm{c}$ receptors that facilitate factor $\mathrm{c}$ activation; and by releasing tissue-type plasminogen activator (tPA) (13). However, COVID-19 disrupts these processes and damages endothelial cells via direct viral toxicity and immune-mediated inflammatory changes (14-17). In a 2020 Lancet study, Varga et al provided histological evidence showing the presence of viral bodies within endothelial cells as well as the presence of accumulated of inflammatory cells with evidence of endothelial and inflammatory cell death (17). The authors concluded that COVID-19 infection resulted in endotheliitis in multiple organs throughout the body. This finding is supported by other studies reporting severe endothelial damage in pathology specimens from both fatal and non-fatal cases of COVID-19 (14). COVID-19 has also been directly shown to infect engineered human blood vessel endothelial cells in vitro (18).

Njoum et al evaluated the sensitivity of $P P G_{A C}$ and $P P G_{D C}$ components to changes in hemorheology following changes in endovascular shear rates and induced clotting in vivo (6). The authors reported that $P P G_{A C}$ amplitude started to decrease within a few seconds of administering clotting agent and continued to drop until the blood achieved maximum clotting after approximately 20 minutes. The PPG $A C$ waveform at that moment displayed significantly lower amplitudes and disrupted $\mathrm{PPG}_{\mathrm{AC}}$ morphology (6). These findings can be explained by the presence of a fibrinous matrix, which alters the absorption and scattering properties of the sample. Similar PPG changes have also been evaluated in the context of sympathetic blockade (19-21), thermal stress (22), sleep (23,24), and altered venous oxygenation (25). Recent investigations have reported that PPG amplitude drops during sleep are an independent marker for cardiometabolic outcomes (24) and hypertension (25).

Given the current body of evidence surrounding hemorheological changes and reduced PPG amplitude, it is unsurprising that patients with COVID-19 experienced increased rates of PPG dropout. Of the 197 enrolled ICU patient with COVID-19, every patient exhibited at least one PPG dropout event during PPG recording $(n=197, p<0.05)$. Furthermore, within COVID-19-positive PPG tracings, the median PPG dropout rate was 0.58 (median 0.58 , IQR $0.42-0.72, \mathrm{p}<0.05$ ). This indicates that roughly $58 \%$ of individual pulse waves within the cumulative PPG tracings of all 197 patients translated to a PPG dropout event. PPG dropout was not only displayed by every included COVID-19 patient but displayed to a considerable extent.

In contrast, non-COVID patients had low rates of PPG dropout with a median PPG dropout rate of 0.0 (median 0.0; IQR 0.0-0.0, p<0.05). Because non-COVID control patients underwent PPG recording prior to the arrival of COVID-19 to the United States, these patients were assumed to be without COVID-19-related inflammatory or hypercoagulable processes. It was therefore expected that these patients would produce PPG tracings without amplitude reductions. However, because these control patients were retrospectively selected using computer randomization, it was impossible to control for all confounding factors. Among non-COVID control patients, seven patients or $2.3 \%$ of controls displayed one or more PPG dropout event. 
The events observed in the control population may be attributable to artifact or may be the result of confounding underlying disease processes.

The lowest PPG dropout rate recorded among patients with COVID-19 was 0.11, and the highest PPG dropout rate recorded among patients without COVID-19 was 0.01. Even the PPG dropout rate for the lowest outlier among patients with COVID-19 is higher than the highest reported PPG dropout rate displayed among patients without COVID-19 by more than a factor of ten. This suggests that PPG dropout rate differs strongly between the two groups and could potentially be used to differentiate patients with COVID-19 from patients without COVID-19. However, further investigation into the sensitivity and specificity of PPG dropout as a screening tool for COVID-19 is needed. PPG dropout may not be specific to COVID-19 and evaluations of possible PPG changes in the context of sepsis, cardiovascular disease, and similar inflammatory processes are also warranted.

PPG dropout rate also correlated closely with normalized D-dimer trends. COVID-19 results in increased arterial, venous, and microvascular thrombus formation (5,26-27). Further, despite the frequent use of prophylactic and therapeutic anticoagulation, patients with COVID-19 display similar thrombin generation to healthy controls (7). D-dimer is therefore a well-described biophysical marker used for following COVID19 disease severity and prognosis $(7,8)$. Xiaokang et al evaluated of the value of D-dimer as a tool for COVID-19 prognosis in 1,114 patients with COVID-19 from Wuhan, China (8). The authors reported that Ddimer levels were closely related to COVID-19 prognosis and that D-dimer levels were more likely to be elevated among severely ill patients than patients with mild disease (8). Xiaokang et al also reported that D-dimer levels were significantly higher among patients who died from COVID-19 than those who survived and that the optimal probability cutoff value for D-dimer was $2.025 \mathrm{mg} / \mathrm{L}$ (8). Cummings et al conducted a similar investigation in New York City and concluded that D-dimer is an independent risk factor for in-hospital mortality (28). Strong correlation between D-dimer levels and PPG dropout rates following linear regression $\left[R^{2}=0.919(p<0.05)\right]$, therefore supports the use of PPG dropout rate as a possible COVID-19 monitoring tool.

\section{Limitations}

This study has several limitations including sample size and the use of retrospectively collected control patients. Recruitment was limited by the ability of a single clinical research coordinator to obtain armband PPG recordings for all included patients and for physician investigators to review relevant electronic medical records. Sample size was generally adequate, but this limitation became apparent during comparison of PPG dropout and D-dimer level trends. A minimum of seven sequential daily Ddimer levels was required to establish a serial D-dimer and PPG dropout trend. Therefore, all patients who became discharged or deceased within seven days were excluded from that portion of the analysis, leaving only 21 included patients. However, we chose to include all available data from all 197 patients with COVID-19 irrespective of the duration of stay to complete a scatter plot comparison of serum d-dimer values with PPG dropout rate and linear regression demonstrated a strong correlation. 
Because the control population was randomly and retrospectively collected, we did not select a matched population for comparison. However, the goal was to identify a population that reflected the general population of patients treated within our institution and the two cohorts were similar in terms of age, sex, ethnicity, and comorbidities.

\section{Conclusion}

Changes in PPG amplitude were previously described in vitro following artificially induced clotting activation. Within the present study, analysis of PPG waveforms from 197 patients with COVID-19 (a population with well-described hypercoagulability and pro-inflammatory processes) vs. 300 non-COVID controls successfully identified increases in PPG dropout rates among patients with COVID-19, indicating that PPG dropout rate changes can also be found in vivo. PPG dropout rates were significantly higher among patients with COVID-19 than patients without COVID-19 and closely mirrored trends in serum ddimer elevation. PPG dropout rate may be a useful addition to existing COVID-19 monitoring. PPG monitoring has the added benefits of being non-invasive, painless, and dynamic (offering results in realtime). PPG can be placed on a patient continuously for monitoring of evolving clinical states and can also be used remotely, without increasing disease exposure. However, inflammatory changes and hypercoagulability are not unique to COVID-19 and further investigations should evaluate the sensitivity and specificity of PPG dropout for COVID-19 and investigate the presence of PPG dropout during other inflammatory disease processes.

\section{Declarations}

\section{Author contributions:}

JP, MW, HW, FH, SL, and SHW contributed to the conceptualization of this manuscript. SH, FH, and HW contributed to data collection. MW and HW contributed to data analysis. JP, SL, MW, HW, and HW contributed to data interpretation. JP, MW, and HW drafted and revised the text. All authors reviewed the text and approve of it in its final form.

\section{Ethical Consideration:}

This study was approved by the Mount Sinai Medical Center (MSMC) Institutional Review Board.

\section{Conflicts of Interest and Funding:}

Jacquelin Peck, MD; Stephen Lee, MD; and Frederick Hasty, MD have no conflicts of interest to declare. 
The photoplethysmography device used in this study (TigerTech CovidPlus Monitor ${ }^{\mathrm{T} M}$ ) was provided by TigerTech Solutions Inc.. Stephanie Hendricks, BA is a research coordinator employed by Tiger Tech Solutions, Inc. Michael Wishon, PhD is Head of Data Sciences and Analytics for Tiger Tech Solutions, Inc. S. Howard Wittels is Chief Medical Officer for Tiger Tech Solutions, Inc. Harrison Wittels, Esq. is Chief Executive Officer for Tiger Tech Solutions, Inc.

\section{Acknowledgements:}

The Tiger Tech Warfighter Monitor (TM) was developed partially under a SBIR contract (W81XWH19C0059) from the Defense Health Agency. However, no funding was provided for the present post-development study using the device.

\section{References}

1. Piagnerelli M, Boudjeltia KZ, Vanhaeverbeek M, Vincent JL. Red blood cell rheology in sepsis. Intensive Care Med. 2003;29(7):1052-1061. doi:10.1007/s00134-003-1783-2.

2. "WHO Coronavirus (COVID-19) Dashboard." World Health Organization, World Health Organization, covid19.who.int/.

3. Maier CL, Truong AD, Auld SC, Polly DM, Tanksley CL, Duncan A. COVID-19-associated hyperviscosity: a link between inflammation and thrombophilia?. Lancet. 2020;395(10239):17581759. doi:10.1016/S0140-6736(20)31209-5.

4. Ranucci M, Ballotta A, Di Dedda U, et al. The procoagulant pattern of patients with COVID-19 acute respiratory distress syndrome. J Thromb Haemost. 2020;18(7):1747-1751. doi:10.1111/jth.14854.

5. Oxley TJ, Mocco J, Majidi S, et al. Large-Vessel Stroke as a Presenting Feature of Covid-19 in the Young. N Engl J Med. 2020;382(20):e60. doi:10.1056/NEJMc2009787.

6. Njoum H, Kyriacou PA. Photoplethysmography for the Assessment of Haemorheology. Sci Rep. 2017;7(1):1406. Published 2017 May 3. doi:10.1038/s41598-017-01636-0.

7. de la Morena-Barrio ME, Bravo-Pérez C, Miñano A, et al. Prognostic value of thrombin generation parameters in hospitalized COVID-19 patients. Sci Rep. 2021;11(1):7792. Published 2021 Apr 8. doi:10.1038/s41598-021-85906-y.

8. He X, Yao F, Chen J, et al. The poor prognosis and influencing factors of high D-dimer levels for COVID-19 patients. Sci Rep. 2021;11(1):1830. Published 2021 Jan 19. doi:10.1038/s41598-02181300-w

9. Office of the Commissioner. "Coronavirus (Covid-19) UPDATE: FDA Authorizes First Machine LEARNING-BASED Screening Device to Identify Certain Biomarkers That May Indicate COVID-19 Infection." U.S. Food and Drug Administration, FDA, 19 Mar. 2021, www.fda.gov/news-events/pressannouncements/coronavirus-covid-19-update-fda-authorizes-first-machine-learning-based-screeningdevice-identify. 
10. Holshue ML, DeBolt C, Lindquist S, et al. First Case of 2019 Novel Coronavirus in the United States. $N$ Engl J Med. 2020;382(10):929-936. doi:10.1056/NEJMoa2001191.

11. Smith, Steven W. The Scientist and Engineer's Guide to Digital Signal Processing. California Technical Publ, 1999.

12. Ferrario $\mathrm{CM}$, Jessup J, Chappell MC, et al. Effect of angiotensin-converting enzyme inhibition and angiotensin II receptor blockers on cardiac angiotensin-converting enzyme 2. Circulation. 2005;111(20):2605-2610. doi:10.1161/CIRCULATIONAHA.104.510461.

13. Ito T, Kakuuchi M, Maruyama I. Endotheliopathy in septic conditions: mechanistic insight into intravascular coagulation. Crit Care. 2021;25(1):95. Published 2021 Mar 8. doi:10.1186/s13054-02103524-6

14. Ackermann M, Verleden SE, Kuehnel M, et al. Pulmonary Vascular Endothelialitis, Thrombosis, and Angiogenesis in Covid-19. N Engl J Med. 2020;383(2):120-128. doi:10.1056/NEJMoa2015432

15. McGonagle D, O'Donnell JS, Sharif K, Emery P, Bridgewood C. Immune mechanisms of pulmonary intravascular coagulopathy in COVID-19 pneumonia. Lancet Rheumatol. 2020;2(7):e437-e445. doi:10.1016/S2665-9913(20)30121-1

16. Goshua G, Pine AB, Meizlish ML, et al. Endotheliopathy in COVID-19-associated coagulopathy: evidence from a single-centre, cross-sectional study. Lancet Haematol. 2020;7(8):e575-e582. doi:10.1016/S2352-3026(20)30216-7.

17. Varga Z, Flammer AJ, Steiger $P$, et al. Endothelial cell infection and endotheliitis in COVID-19. Lancet. 2020;395(10234):1417-1418. doi:10.1016/S0140-6736(20)30937-5

18. Monteil V, Kwon H, Prado P, et al. Inhibition of SARS-CoV-2 Infections in Engineered Human Tissues Using Clinical-Grade Soluble Human ACE2. Cell. 2020;181(4):905-913.e7. doi:10.1016/j.cell.2020.04.004.

19. Betta M, Handjaras G, Ricciardi E, et al. Quantifying peripheral sympathetic activations during sleep by means of an automatic method for pulse wave amplitude drop detection. Sleep Med. 2020;69:220-232. doi:10.1016/j.sleep.2019.12.030

20. Mihaljevic S, Bevanda M, Reiner K, Sporcic K, Mihaljevic L, Cacic M. Area Under the Curve of Finger Photoplethysmography as an Evaluation Measure for Sympathetic Activity During Lumbar Epidural Anaesthesia. Turk J Anaesthesiol Reanim. 2018;46(2):147-150. doi:10.5152/TJAR.2018.56688

21. Babchenko A, Davidson E, Ginosar Y, et al. Photoplethysmographic measurement of changes in total and pulsatile tissue blood volume, following sympathetic blockade. Physio/ Meas. 2001;22(2):389396. doi:10.1088/0967-3334/22/2/310

22. Budidha K, Kyriacou PA. In vivo investigation of ear canal pulse oximetry during hypothermia. J Clin Monit Comput. 2018;32(1):97-107. doi:10.1007/s10877-017-9975-4.

23. Abay TY, Kyriacou PA. Reflectance Photoplethysmography as Noninvasive Monitoring of Tissue Blood Perfusion. IEEE Trans Biomed Eng. 2015;62(9):2187-2195. doi:10.1109/TBME.2015.2417863.

24. Hirotsu C, Betta M, Bernardi G, et al. Pulse wave amplitude drops during sleep: clinical significance and characteristics in a general population sample. Sleep. 2020;43(7):zsz322. 
doi:10.1093/sleep/zsz322

25. Zou D, Grote L, Radlinski J, Eder DN, Lindblad U, Hedner J. Nocturnal pulse wave attenuation is associated with office blood pressure in a population based cohort. Sleep Med. 2009;10(8):836-843. doi:10.1016/j.sleep.2008.10.001

26. Wool GD, Miller JL. The Impact of COVID-19 Disease on Platelets and Coagulation. Pathobiology. 2021;88(1):15-27. doi:10.1159/000512007

27. Kashi M, Jacquin A, Dakhil B, et al. Severe arterial thrombosis associated with Covid-19 infection. Thromb Res. 2020;192:75-77. doi:10.1016/j.thromres.2020.05.025

28. Cummings MJ, Baldwin MR, Abrams D, et al. Epidemiology, clinical course, and outcomes of critically ill adults with COVID-19 in New York City: a prospective cohort study. Lancet. 2020;395(10239):17631770. doi:10.1016/S0140-6736(20)31189-2

\section{Figures}

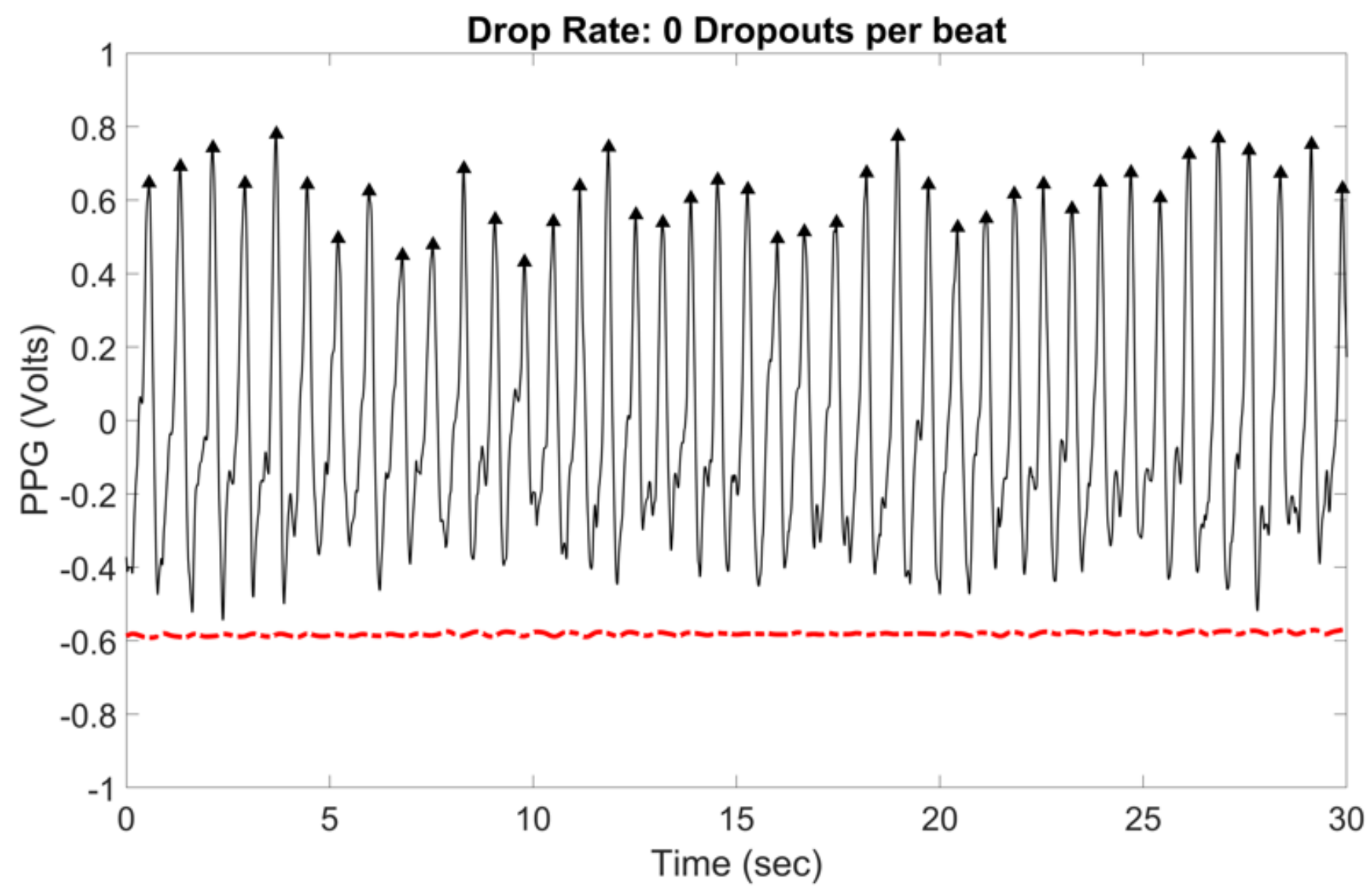

Figure 1

Representative photoplethysmography tracing from a non-COVID patient. *The black solid line demonstrates a sample PPG waveform taken from a non-COVID patient. The bias was removed, and the 
PPG was normalized for clarity. Black triangles represent detected pulse wave peaks utilized for calculating pulse rate. The red dotdash line marks predetermined PPG mean minus standard deviation. There are no PPG dropouts (excursions below the dotdash line) within this sample.

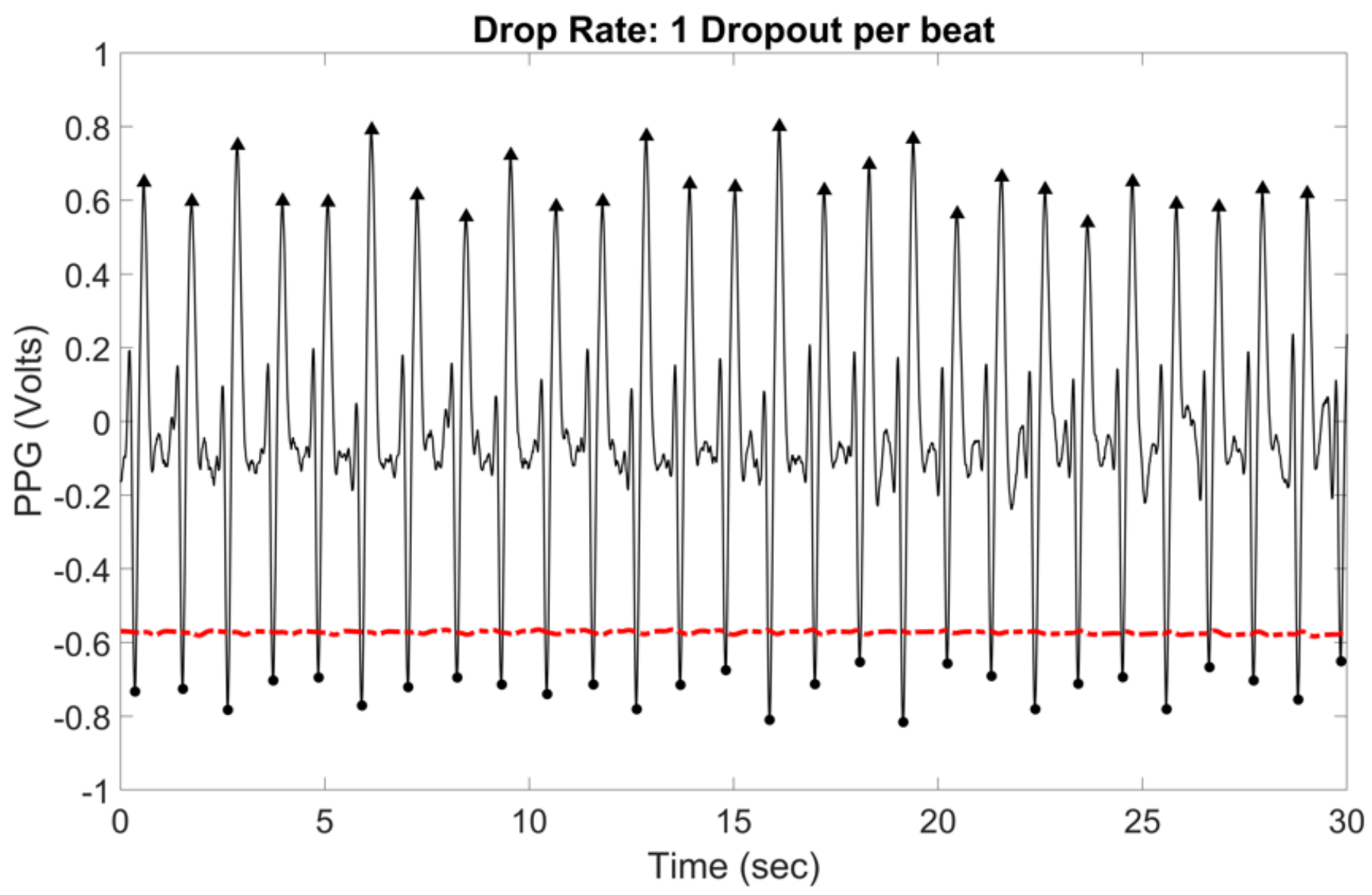

Figure 2

Photoplethysmography tracing from a COVID-19 Positive patient. *The black solid line demonstrates a sample PPG waveform taken from a COVID-19-POSITIVE patient. The bias was removed, and the PPG was normalized for clarity. Black triangles represent detected pulse wave peaks utilized for calculating pulse rate. The red dotdash line marks predetermined PPG mean minus standard deviation. Each pulse wave results in a PPG dropout (excursions below the dotdash line) within this sample. 


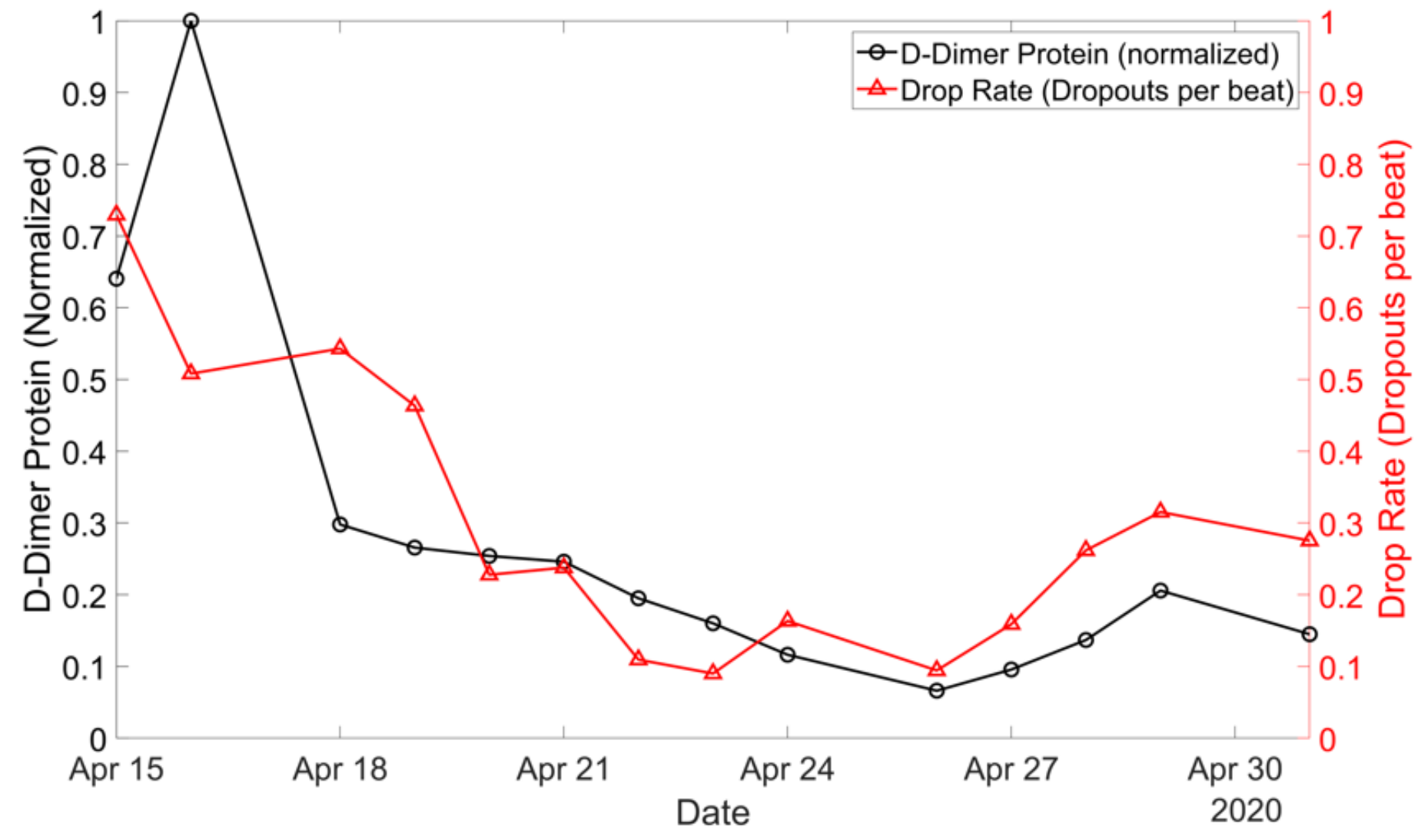

Figure 3

Serum D-Dimer Protein Level and PPG Dropout Rate Plotted over Time in a COVID-19 Patient. *Trends in serum d-dimer and drop rate plotted over time for a sample COVID-19 patient. The black line denotes normalized serum d-dimer levels ( $d$-dimer normalized by dividing all the levels by the maximum D-Dimer level acquired for that patient during their stay) and the red line denotes PPG dropout rate (normalized by 1.65 with $\mathrm{DC}$ bias was removed). 


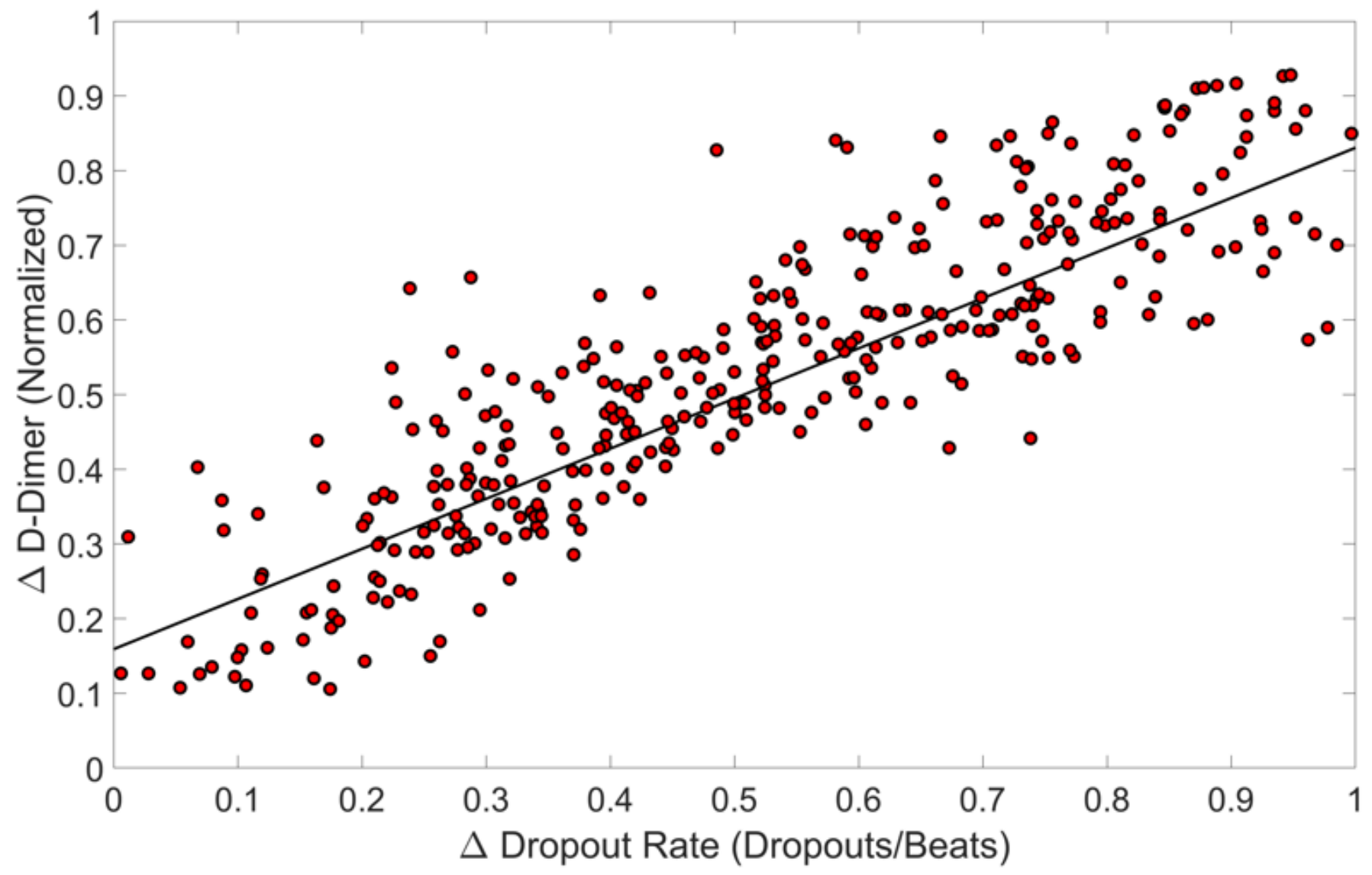

Figure 4

Plot of D-Dimer vs. PPG Dropout Rate for 197 COVID-19 Patients. *Scatter plot depicting change in normalized serum d-dimer vs. change in drop rate. There is one dot for each of the 197 COVID-19 patients. The y-axis denotes the change in normalized serum d-dimer level during hospitalization (maximum d-dimer level minus minimum d-dimer level divided by maximum $\mathrm{d}$-dimer level) and the $\mathrm{x}$-axis denotes the corresponding change in PPG dropout rate from the date of maximum d-dimer to the date of minimum d-dimer (PPG dropout rate from date of maximum d-dimer level minus the PPG dropout rate from the date of the minimum d-dimer) (PPG dropout rate was, again, normalized by 1.65 with DC bias was removed). ${ }^{\star \star}$ Solid line marks line of best fit. $R 2=0.919(p<0.05)$ 